\title{
Tratamentos para obtenção de TaC em superfície de grafite. Parte III: Imersão em Suspensão Aquosa de Ta e $\mathrm{Ta}_{2} \mathrm{O}_{5}$
}

\section{(TaC formation on graphite surface. \\ Part III: Immersion in Aqueous Suspension of Ta and $\mathrm{Ta}_{2} \mathrm{O}_{5}$ )}

\author{
H. J. Izário Fo 1, F. Vernilli Jr. ${ }^{2}$, D. V. B. S. Pinto ${ }^{3}$, N. Baccan ${ }^{4}$, A. F. Sartori ${ }^{2}$ \\ ${ }^{1}$ Departamento de Engenharia Química - FAENQUIL \\ ${ }^{2}$ Departamento de Engenharia de Materiais - FAENQUIL \\ C. P. 116, 12600-000,Lorena, SP. \\ vernilli@demar.faenquil.br \\ ${ }^{3}$ Instituto de Química da Universidade de S. Paulo - USP \\ ${ }^{4}$ Instituto de Química da Universidade de Campinas - UNICAMP
}

\begin{abstract}
Resumo
Este trabalho apresenta uma nova metodologia para a formação de uma camada de carbeto de tântalo em superfície de grafite, que consiste da imersão dos tubos de grafite em suspensões aquosas de tântalo e de óxido de tântalo, com posterior tratamento térmico a $2100^{\circ} \mathrm{C}$. Para facilitar a aderência dos compostos de tântalo sobre a superfície do tubo, utilizou-se álcool polivinílico (PVOH). Para obter uma camada de carbeto de tântalo homogênea e aderente, os parâmetros concentração das suspensões e temperatura dos corpos de prova antes da imersão, também foram analisados. A camada de carbeto de tântalo formada foi caracterizada utilizando microscopia eletrônica de varredura (MEV) e difratometria de raios $\mathrm{X}$.
\end{abstract}

Palavras-chave: carbeto de tântalo, eletrodeposição, fluoretos fundidos, grafite.

\section{INTRODUÇÃO}

Quando se trata de análise de metais refratários utilizando espectrometria de absorção atômica com atomização eletrotérmica em forno de grafite a altas temperaturas, pode ocorrer a formação de carbeto entre o metal majoritário e o material do tubo, acarretando inúmeras interferências. Desta forma, para se determinar as impurezas metálicas de interesse na presença da matriz de um elemento cuja cinética química tende a formar carbeto metálico, é necessário realizar um tratamento superficial nos tubos de grafite de maneira adequada, para que não haja interação química entre o grafite do tubo e o elemento majoritário da matriz analisada [1-3].

Neste trabalho é apresentada uma nova metodologia, que consiste da imersão de corpos de prova de grafite em suspensões de tântalo

\begin{abstract}
(20)
Abstract

This work presents the methodology studied for the formation of a tantalum carbide layer in a graphite surface. The methodology consists of the immersion of the graphite tubes in aqueous suspensions of tantalum and tantalum oxide, with subsequent thermal treatment at $2100^{\circ}$ C. Poly (vinyl alcohol - PVOH) was used in order to facilitate the adherence of the tantalum composite on the tube surface. Parameters such as concentration and temperature of the suspension and the temperature and atmosphere of the thermal treatment were investigated in order to obtain an homogeneous and adherent layer of tantalum carbide. The tantalum carbide layer was characterized using scanning electron microscopy and analysis of phases by X-ray diffraction.
\end{abstract}

Keywords: electrodeposition, graphite, tantalum carbide.

e de óxido de tântalo. A utilização de suspensão permite avaliar, após tratamento térmico, a camada de carbeto de tântalo formada sob diferentes condições experimentais, a fim de avaliar a metodologia para utiliza-la no tratamento superficial de tubos de grafite.

A eficiência da metodologia estudada foi avaliada pela aderência e homogeneidade da camada de carbeto de tântalo formada, monitorada por microscopia eletrônica de varredura; a formação do carbeto de tântalo após tratamento térmico foi confirmada por difratometria de raios $\mathrm{X}$.

\section{EXPERIMENTAL}

Para este estudo foram utilizados como corpos de prova, tubos de grafite sem revestimento pirolítico com $6 \mathrm{~mm}$ de diâmetro interno, 
$28 \mathrm{~mm}$ de comprimento e com $1 \mathrm{~mm}$ de espessura de parede fornecidos pela Perkin Elmer, a Fig. 1 apresenta a imagem da microestrutura superficial do tubo [4]. Todos os reagentes utilizados neste trabalho foram de pureza analítica (P.A.).

$\mathrm{Na}$ preparação das suspensões de óxido de tântalo em meio aquoso, utilizou-se o álcool polivinílico $(\mathrm{PVOH})$ para facilitar a aderência do óxido metálico sobre a superfície do tubo, melhorando a trabalhabilidade antes do tratamento térmico; como a viscosidade da suspensão foi alterada com a adição de PVOH , este também funcionou como um agente suspensor na suspensão de tântalo metálico [5-7].

As suspensões para os ensaios de impregnação por imersão foram preparadas em solução aquosa de álcool polivinílico, com concentrações de sólidos de 15 e $50 \% \mathrm{~m} / \mathrm{v}$ para o óxido de tântalo e $25 \% \mathrm{~m} / \mathrm{v}$ para o tântalo metálico.

Os tubos de grafite foram imersos na suspensão à temperatura ambiente $\left(25^{\circ} \mathrm{C}\right)$ e, também, aquecidos a $80^{\circ} \mathrm{C}$ antes da imersão. As impregnações foram realizadas por poucos segundos sendo, o tudo retirado por meio de uma pinça e seco com ar quente $\mathrm{a} \approx 50{ }^{\circ} \mathrm{C}$.

Os tratamentos térmicos dos tubos revestidos através desta metodologia foram realizados em forno tripolar, o qual possui elemento resistivo de tântalo no formato de uma barca de $\approx 1 / 2$ " $\mathrm{x} 1$ " x 5", que também funciona como o porta amostra, o aquecimento é controlado por reostato e a leitura da temperatura é realizada com pirômetro ótico. A atmosfera de trabalho foi argônio em $1 \times 10^{-8}$ $\mathrm{MPa}$. O ciclo dos tratamentos térmicos realizados estão apresentados na Tabela I.

A formação da camada de carbeto de tântalo foi confirmada por análise de difratometria de raios $\mathrm{X}$; a homogeneidade e morfologia dos grãos, foram avaliadas através de microscopia eletrônica de varredura.

Para realizar as análises descritas, os tubos tratados termicamente foram cortados longitudinalmente e, posteriormente, transversalmente.

\section{RESULTADOS E DISCUSSÃO}

O tratamento de superfície do tubo de grafite realizado, utilizando suspensões de $\mathrm{Ta}_{2} \mathrm{O}_{5}$ em solução aquosa de álcool polivinilico, forneceu tubos que apresentaram macroscopicamente

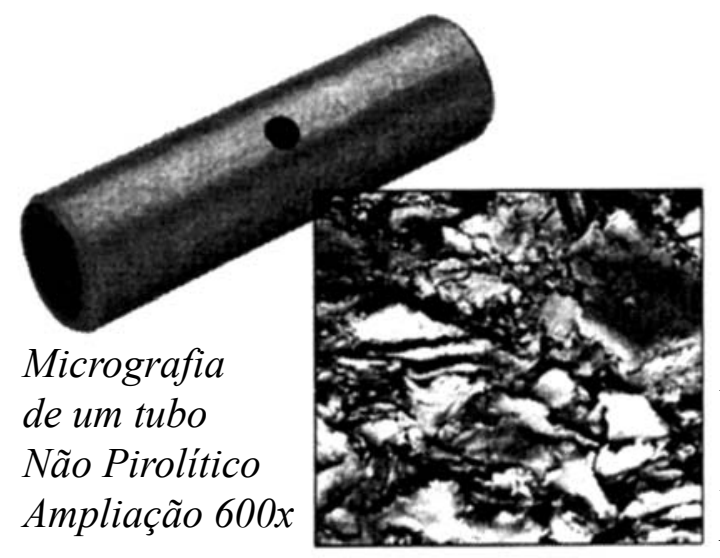

Figura 1: Tubos de grafite fornecidos pela Perkin Elmer [2]. [Figure 1: Graphite tubes by Perkin Elmer [2].] recobrimento em toda a superfície e boa aderência da camada.

A Fig. 2 apresenta as micrografias de uma das amostras imergidas na suspensão de $\mathrm{Ta}_{2} \mathrm{O}_{3}$ com $15 \%$ de concentração de sólidos, à temperatura ambiente $\left(25^{\circ} \mathrm{C}\right)$. Na Fig. 2 a pode-se observar

(a)

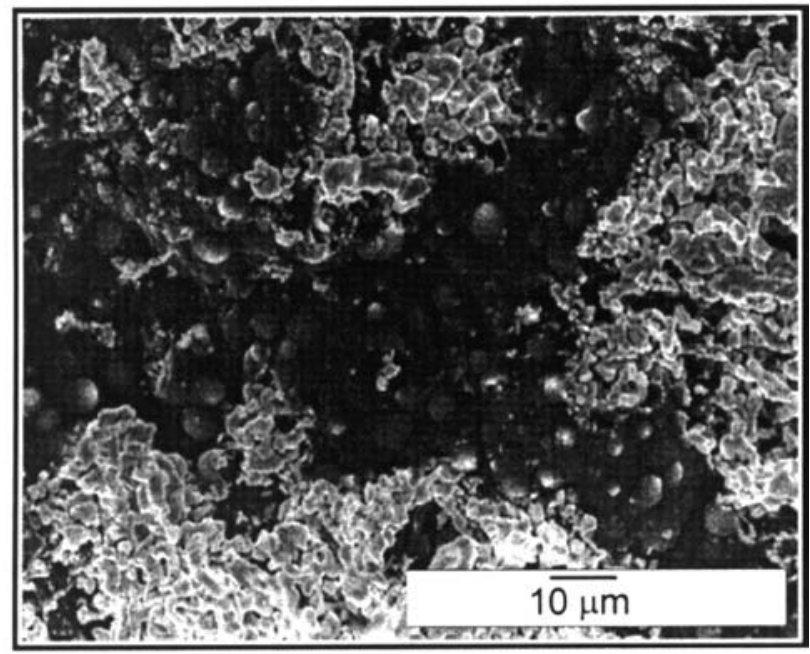

(b)

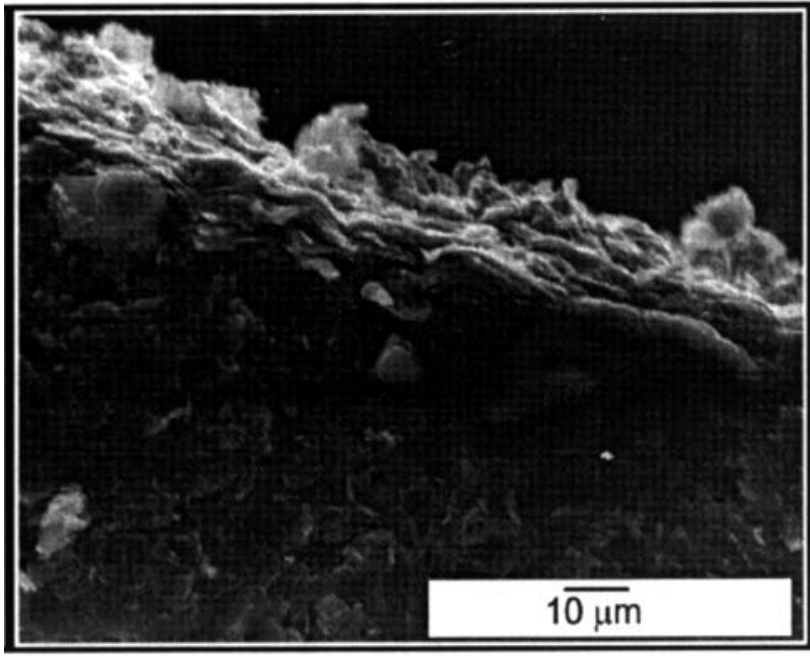

Figura 2: Micrografias da camada de carbeto de tântalo obtido por imersão em suspensão de $15 \% \mathrm{~m} / \mathrm{v} \mathrm{Ta}_{2} \mathrm{O}_{5}$ : (a) Superfície interna do tubo e (b) Seção transversal do tubo.

[Figure 2: Micrographs of the tantalum carbide layer obtained by immersion in suspension of $15 \% \mathrm{w} / \mathrm{v} \mathrm{Ta} \mathrm{O}_{5}$ : (a) internal surface of the tube and (b) crosssection of the tube. ]

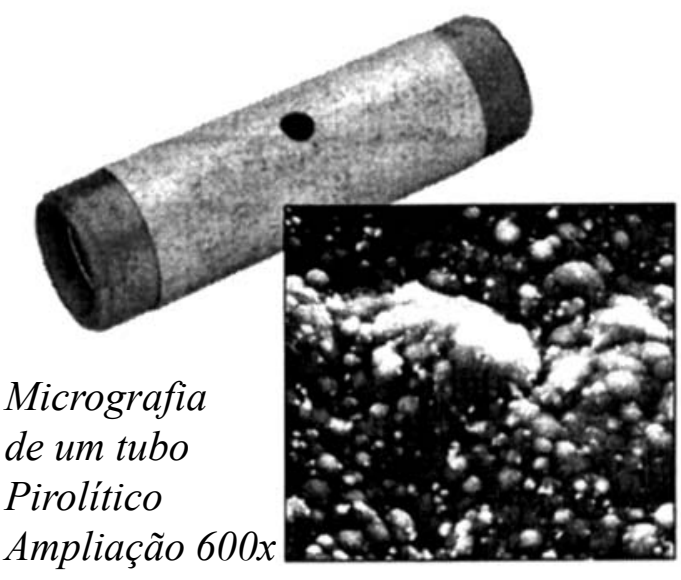


(a)

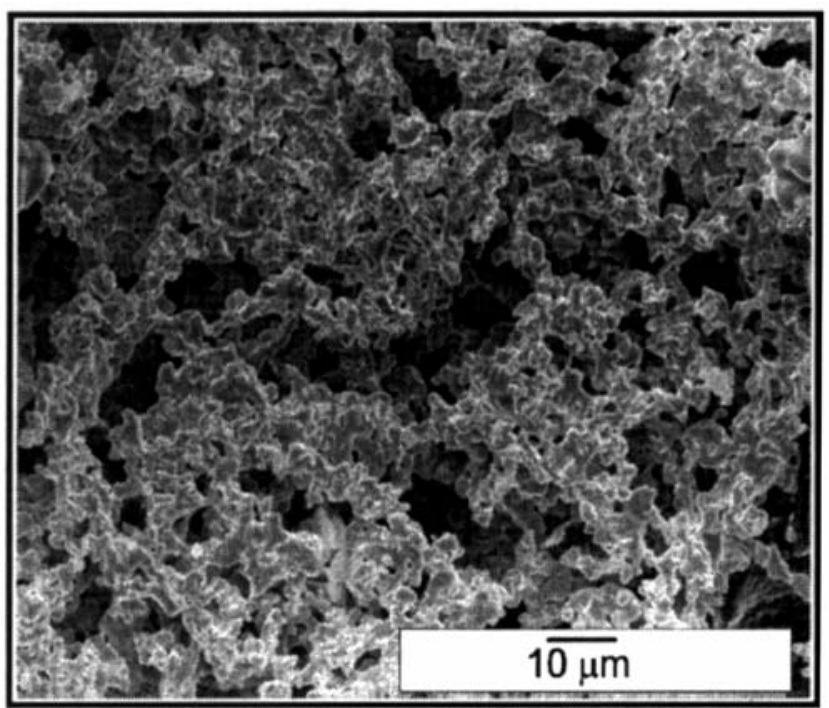

(b)

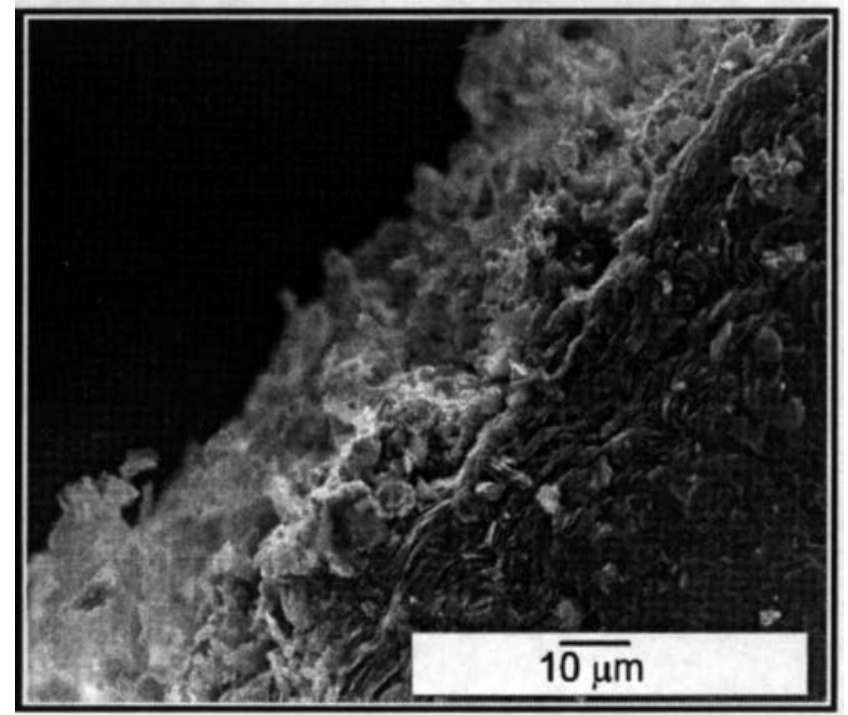

Figura 3: Micrografias da camada de carbeto de tântalo obtida por imersão em suspensão de $50 \% \mathrm{~m} / \mathrm{v}$ de $\mathrm{Ta}_{2} \mathrm{O}_{5}$ : (a) superfície interna do tubo e (b) seção transversal do tubo.

[Figure 3: Micrographs of the tantalum carbide layer obtained by immersion in suspension of $50 \% \mathrm{w} / \mathrm{v}$ of $\mathrm{Ta}_{2} \mathrm{O}_{5}:$ (a) interns surface of the tube and (b) cross-section of the tube.]

duas camadas, uma morfologia nodular, na camada inferior e uma morfologia irregular, na camada superior, isto pode ser atribuído ao processo de formação da camada, que ocorreu por capilaridade, com isso as camadas mais externas tendem a uma maior porosidade. Na Fig. 1 b pode-se observar a seção transversal da camada de carbeto de tântalo formada, a qual apresenta uma formação em placas. A determinação da espessura da camada de $\mathrm{TaC}$ formada ficou prejudicada, pois como a interface entre as duas camadas ( $\mathrm{TaC}$ e grafite) não é nítida, isto devido principalmente a dificuldade em se preparar a superfície da amostra para observação microscópica, em função da grande diferença entre as durezas deste dois materiais.

A Fig. 3 apresenta as micrografias das amostras imergidas na suspensão de $50 \%$ de concentração de óxido de tântalo, à temperatura ambiente. Na Fig. 3a pode-se observar somente a (a)

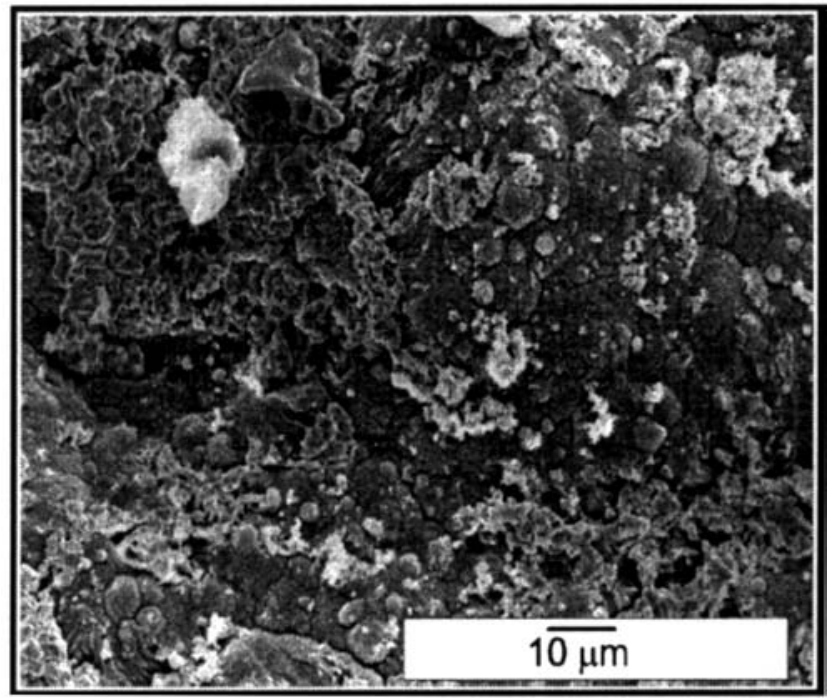

(b)

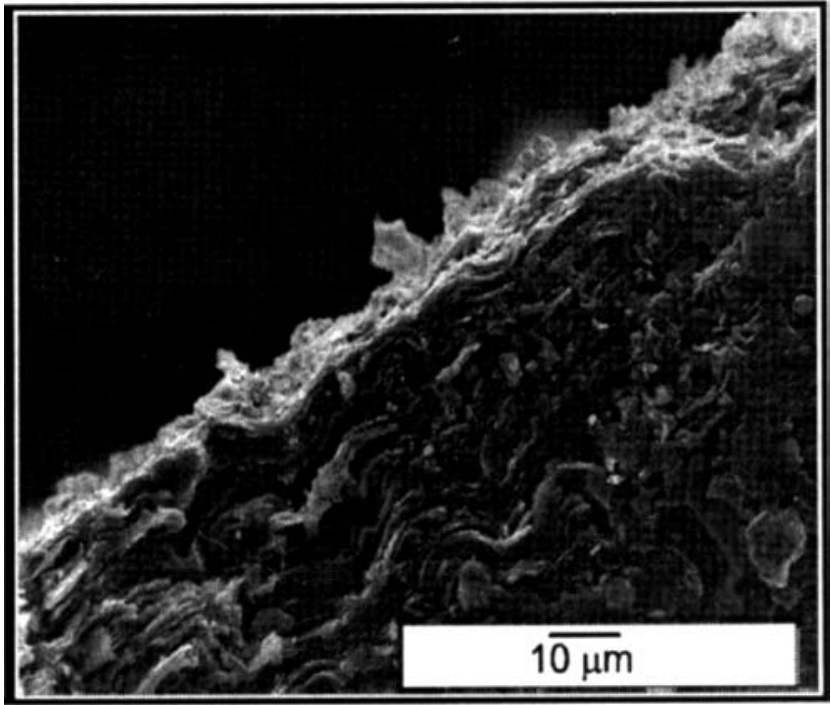

Figura 4: Micrografias da camada de carbeto de tântalo obtida por imersão em suspensão de $\mathrm{Ta}_{2} \mathrm{O}_{5}$, com aquecimento prévio do tubo: (a) superfície interna do tubo e (b) seção transversal do tubo.

[Figure 4: Micrographs of the tantalum carbide layer obtained by immersion in suspension of $\mathrm{Ta}_{2} \mathrm{O}_{5}$, with previous heating of the tube: (a) internal surface of the tube and (b) cross-section of the tube.]

camada superior com uma morfologia irregular e não compacta; na Fig. 3 b pode-se observar a seção transversal das camadas de carbeto formadas, a porosidade apresentada na camada superior poderá ser prejudicial à performance analítica do tubo.

As micrografias de uma das amostras preparadas aquecendose o tubo com ar quente, antes da imersão em suspensões de $\mathrm{Ta}_{2} \mathrm{O}_{3}$ com $15 \% \mathrm{~m} / \mathrm{v}$, são apresentadas na Fig. 4, onde pode-se observar (Fig. 4a) que a camada de carbeto de tântalo formada é compacta, apresentando grandes aglomerados de grãos nodulares e na Fig. 4b pode-se observar a seção transversal da camada de carbeto formada. Este procedimento apresentou melhores resultados em relação aos outros procedimentos sem aquecimento prévio do tubo, o que comprova que a temperatura ajuda a permeabilizar o grafite da superfície do tubo, isto considerando que com o aumento da temperatura ocorreu uma 
(a)

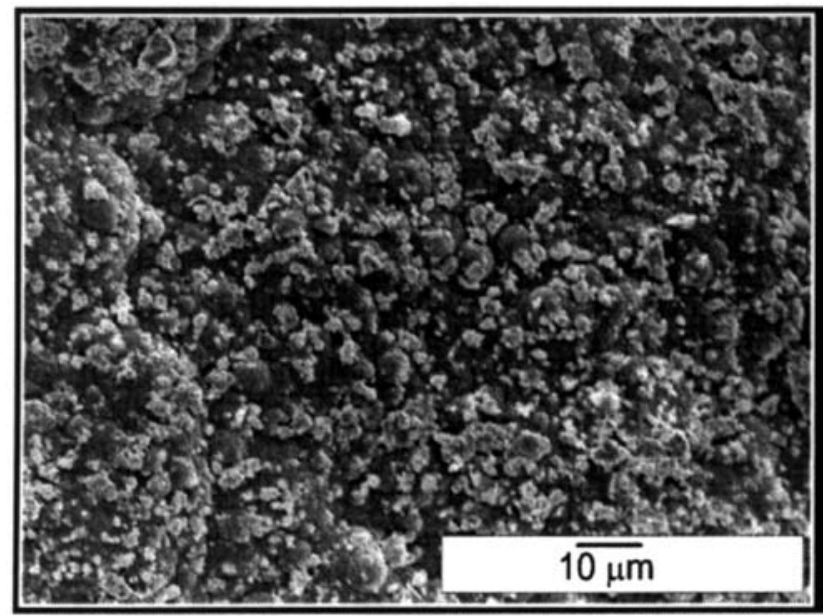

(b)

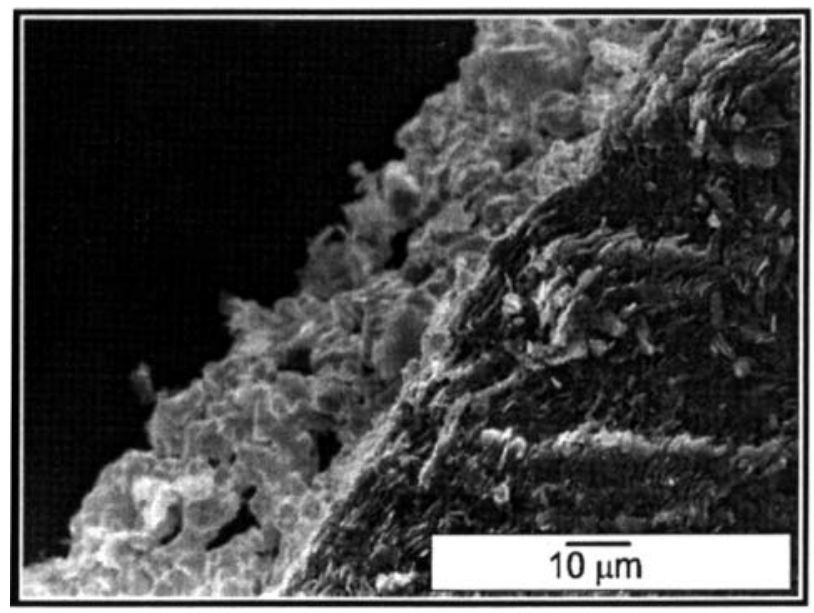

Figura 5: Análise de difração de raios $X$ do carbeto formado pela imersão em suspensão de $\mathrm{Ta}_{2} \mathrm{O}_{5}$ a $15 \% \mathrm{~m} / \mathrm{v}$

Figure 5: Analysis of X rays difraction of the carbide formed by the immersion in suspension of $\mathrm{Ta}_{2} \mathrm{O}_{5}$ to $15 \% \mathrm{~m} / \mathrm{v}$

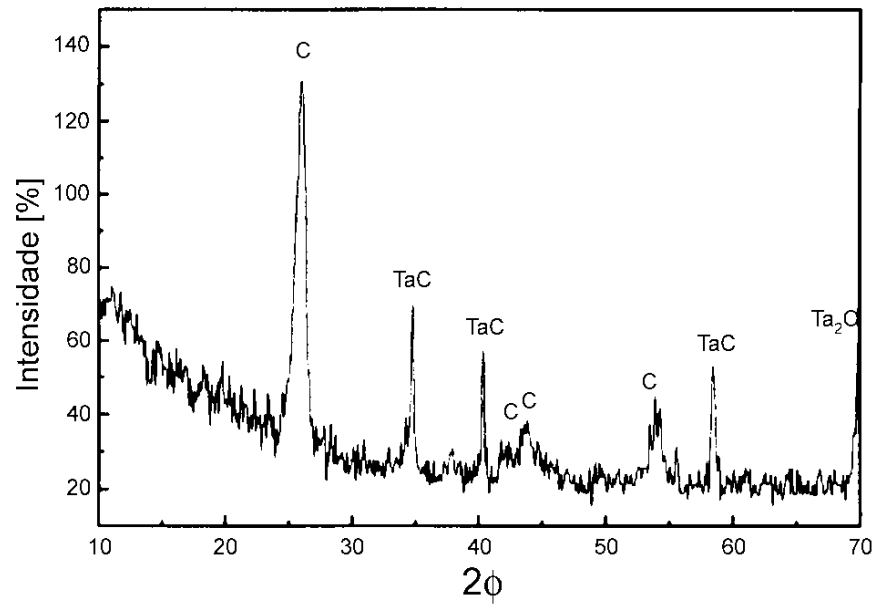

Figura 6: Análise de difração de raios X do carbeto formado pela imersão em suspensão de $\mathrm{Ta}_{2} \mathrm{O}_{5}$ a $15 \% \mathrm{~m} / \mathrm{v}$.

[Figure 6: Analysis of X-rays difraction of the carbide formed by the immersion in suspension of $\mathrm{Ta}_{2} \mathrm{O}_{5}$ to $15 \% \mathrm{w} / \mathrm{v}$.]

diminuição na concentração de oxigênio nos poros, possibilitando a formação de uma camada lisa, densa e homogênea.

As amostras preparadas com suspensão de pó de tântalo metálico apresentaram microestrutura nodular (Fig. 5a); a seção transversal apresentou porosidade (Fig. 5b). Estes resultados não são satisfatórios em relação aos outros anteriormente descritos, apesar de ter-se observado uma superfície homogênea.

A Fig. 6 mostra o resultado da análise de difratometria de raios $\mathrm{X}$ característico do método estudado, onde pode-se confirmar a formação de carbeto de tântalo.

\section{CONCLUSÕES}

Na metodologia de impregnação por imersão em suspensão de óxido de tântalo e de tântalo metálico, observou-se a formação de subcamadas de carbeto de tântalo, que ocasionou o aparecimento de porosidade. Com o aumento da concentração de tântalo na suspensão, a morfologia da camada formada tornou- se mais irregular, demonstrando uma deposição irregular, o que provocou o aumento da porosidade. Estas características físicas da camada de carbeto impossibilitam seu uso analítico, pois alteram a resistividade do tubo, apesar da boa aderência sobre a superfície de grafite e da morfologia nodular nas camadas inferiores do carbeto.

Uma mudança de temperatura no procedimento de preparação do tubo permitiu obter melhores resultados em relação aos procedimentos sem aquecimento prévio do tubo. $\mathrm{O}$ aquecimento do tubo de grafite com ar quente, antes de imergi-lo na suspensão do óxido metálico, possibilitou a formação de uma camada homogênea, lisa e compacta, onde não se detectou a formação de poros. Isto comprova, ainda mais, que o aumento da temperatura permeabiliza a superfície de grafite, isto considerando que com o aumento de temperatura ocorreu uma diminuição na concentração de oxigênio nos poros dos corpos de prova.

Conclui-se, portanto que para aplicações analíticas os tubos de grafite podem ser impregnados por imersão em suspensão de $15 \% \mathrm{~m} / \mathrm{v}$ de $\mathrm{Ta}_{2} \mathrm{O}_{5}$ a $25^{\circ} \mathrm{C}$, com aquecimento prévio do tubo a $\approx$ $80{ }^{\circ} \mathrm{C}$ e tratando termicamente a $2100{ }^{\circ} \mathrm{C}$ em vácuo.

\section{AGRADECIMENTOS}

Os autores agradecem ao Prof. Dr. Mario Cilense (IQ UNESP) e ao Técnico Jorge Luiz Rosa (DEMAR - FAENQUIL).

\section{REFERENCIAS}

[1] J. M. Harnly, "Optimization of electrothermal atomization parameters for simultaneous multielement atomic absorption spectrometry", Anal. Chem. 56, 1 (1984) 48-54.

[2] N. K. Belskii, L. I. Ochertyanova, "Direct determination of impurities in ultrapure tantalum pentoxide by electrothermal atomic absorption spectrometry", J. Anal. Chem. ( USSR ) 48, 12 (1993) 1423-1425.

[3] A. B. Volysky, "Terminology for the modification of graphite tubes with hight-melting carbides used in eletrothermal atomic absorption spectrometry", Spectrochim. Acta 8, 50 (1995) 1417-19. 
[4] Perkin Elmer. Atomic Spectroscopy Supplies Catalog. (1995/ 96).

[5] S. L. Bassner, E. H. Klingenberg "Using poly(vinyl alcohol) as a binder", Am. Ceram. Soc. Bull. 77, 6 (1998) 71-75.

[6] J. Chu, H. Ishibashi, K. Hayashi, H. Takebe, K. Moringa, "Slip casting of continuous functionally gradient material", J. Am. Ceram. Soc. 101, 7 (1993) 841-844

[7] R. A Terpstrap, P. A. C. Pex, A. H. Vries, "Ceramic processing" New York. Chapman \& Hall (1995) 98-99.

(Rec. 26/10/1999, Rev. 03/04/2001, Ac. 20/04/2001) 